\title{
86799 - EDUCAÇÃO EM SAÚDE EM LIGA MULTIPROFISSIONAL DE GERIATRIA E CUIDADOS PALIATIVOS EM 2018: RELATO DE EXPERIÊNCIA DE UM PROJETO EXITOSO
}

\author{
Pôster - Geriatria
}

Jeovana Ceresa / CERESA, J / Acadêmico do Curso de Medicina, Universidade Federal do Rio Grande do Sul; Beatriz Caroline Rodrigues / RODRIGUES, B. C. / Acadêmica do Curso de Psicologia, Universidade Federal do Rio Grande do Sul; Otávio Augusto Gonçalves Dias Cionek / CIONEK, O. A. G. D. / Acadêmico do Curso de Medicina, Universidade Federal do Rio Grande do Sul; Francine da Rocha Flores Giediel Rosa / ROSA, F. R. F. G. / Nutricionista e Pós-Graduanda do Programa de Pós-Graduação em Ciências Médicas: Endocrinologia, Universidade Federal do Rio Grande do Sul; Letícia Guimarães da Silveira / SILVEIRA, L. G. / Acadêmico do Curso de Medicina, Universidade Federal do Rio Grande do Sul; Roberta Rigo Dalla Corte / DALLA CORTE, R. R. / Geriatra, Professora Adjunta do Departamento de Medicina Interna da Universidade Federal do Rio Grande do Sul e docente coordenadora da Liga de Geriatria e Cuidados Paliativos da UFRGS

Introdução: A Liga de Geriatria e Cuidados Paliativos (LiGCP) da Universidade Federal do Rio Grande do Sul é um projeto da Pró-Reitoria de Extensão da UFRGS realizado por um grupo multidisciplinar de alunos da graduação e pós-graduação da UFRGS e coordenado pela professora Roberta Rigo Dalla Corte, do Departamento de Medicina Interna da Faculdade de Medicina da UFRGS. São empreendidas ações em promoção de saúde e educação em Geriatria, Gerontologia e Cuidados Paliativos norteadas ao âmbito acadêmico e público externo mediante exposições de tópicos com palestrantes das áreas. Objetivos: Avaliar o exercício em extensão universitária em educação e promoção de saúde nas três áreas desenvolvido pelo projeto em 2018. Metodologia: Mensurar as atividades de 2018 quanto ao total de eventos, número de inscritos, formação profissional dos inscritos e abrangência da difusão em mídias sociais. Resultados: Foram organizados 7 eventos: a Aula Inaugural, "Cuidados Paliativos: um Olhar Sobre as Necessidades Atuais", "Relação Profissional da Saúde: Uma Relação Essencial”, "Cardiogeriatria: Atualizações em Hipertensão e Dislipidemia", "Nutrição e Cuidados Paliativos, Setembro Dourado: Cuidados Paliativos em Oncologia Pediátrica", "Diabetes e o Paciente Idoso" e "Espiritualidade em Cuidados Paliativos". Ainda que exista o fator restritivo de limitação física do espaço onde são desenvolvidas as ações, na totalidade dos eventos foram contabilizados 749 inscritos. A maior parte dos participantes é da área de Enfermagem (47,6\%), seguido de Medicina (25,87\%), Nutrição (9,1\%), Fonoaudiologia $(6,61 \%)$ e Fisioterapia $(4,86 \%)$, com menores contribuições em número de presentes de graduações de Psicologia, Terapia Ocupacional, Farmácia e Educação. Os eventos foram divulgados na página da Liga no Facebook (fb.me/LiGCPUFRGS), criada em junho de 2015 e que conta com 1.325 seguidores. Agregando a divulgação da totalidade dos eventos, a abrangência foi 12.400 de pessoas alcançadas, 526 reações e 2.877 respostas. Conclusões: Devido à abordagem multidisciplinar objetivada pelo projeto de extensão, há fomento de participação de ouvintes de diferentes formações profissionais 
que enriquecem as discussões do conhecimento científico em Geriatria, Gerontologia e Cuidados Paliativos, bem como o intercâmbio entre graduações da área da Saúde e entre os eixos do tripé universitário de Ensino, Pesquisa e Extensão.

Palavras-chave: Liga Acadêmica, Geriatria, Gerontologia, Cuidados Paliativos. 